\title{
Correction to: Plant Anatomy
}

Correction to:

๑) Springer Nature Switzerland AG 2018

R. Crang et al., Plant Anatomy, https://doi.org/10.1007/978-3-319-77315-5

This book was inadvertently published without updating the following corrections.

1. On page IV, corrected spelling of "Champain" to "Champaign" in the affiliation of Richard Crang.

2. On page 5, third line under heading "Introduction": the following words have been omitted "that are formally not plants, but are protists," to read "algae to bryophytes,".

3. On page 5, sixth line under heading "Introduction": commas have been removed before and after "chlorophyll" to read "pigment chlorophyll and are the primary".

The updated versions of the chapters can be found at https://doi.org/10.1007/978-3-319-77315-5_1 https://doi.org/10.1007/978-3-319-77315-5_2 https://doi.org/10.1007/978-3-319-77315-5_3 https://doi.org/10.1007/978-3-319-77315-5_4 https://doi.org/10.1007/978-3-319-77315-5_5 https://doi.org/10.1007/978-3-319-77315-5_6 https://doi.org/10.1007/978-3-319-77315-5_7 https://doi.org/10.1007/978-3-319-77315-5_8 https://doi.org/10.1007/978-3-319-77315-5_9 https://doi.org/10.1007/978-3-319-77315-5_10 https://doi.org/10.1007/978-3-319-77315-5_11 https://doi.org/10.1007/978-3-319-77315-5_12 https://doi.org/10.1007/978-3-319-77315-5_13 https://doi.org/10.1007/978-3-319-77315-5_14 https://doi.org/10.1007/978-3-319-77315-5_15 https://doi.org/10.1007/978-3-319-77315-5_16 https://doi.org/10.1007/978-3-319-77315-5_17 https://doi.org/10.1007/978-3-319-77315-5_18 https://doi.org/10.1007/978-3-319-77315-5_19 https://doi.org/10.1007/978-3-319-77315-5 
4. On page 5, last line of the first paragraph: word "protists" has been deleted and replaced with "eukaryotes".

5. On page 6, in legend to Figure 1.1a,b: the attributions have been changed from "( $a, b$ RR Wise)" to "(a RR Wise; b public domain)".

6. On page 47, third line under heading "Introduction": "impact in" has been changed to "impact on".

7. On page 55, fifth line in the second paragraph under section 2.7: "objectives lenses" has been changed to "objective lenses".

8. On page 57, fourth line in legend to Figure 2.8: "blue object" has been changed to "orange object".

9. On page 88 , legend to Figure 3.5c-e: “(right)" has been deleted in the second line.

10. On page 90, first paragraph of section 3.5.5: "leaf and" in fifth line has been deleted to read "process of fruit ripening.".

11. On page 90, second paragraph of section 3.5.5: " $\beta$-carotenes" has been changed to "carotenes" in the second line.

12. On page 92, legend to Figure 3.5: two occurrences of "(RR Wise)" in lines 2 and 4 have been deleted and "(n-p RR Wise)" has been retained in the last line of the legend.

13. On page 95, legend to Figure 3.5: "(RR Wise)" has been deleted from the second line and "( $\mathbf{q}, \mathbf{r}$ RR Wise)" has been retained at the end of the legend.

14. On page 98, legend to Figure 3.5: "Scale bar in $(b)=. . . "$ has been changed to "Scale bar in $\mathrm{w}=. .$. ".

15. On page 99, first paragraph of section 3.6 in the fourth line: "on the left side of the figure" has been changed to "in the center of the figure".

16. On page 130, first paragraph of section 4.4: the last sentence "The cell plate has not reached the cell wall on the left but has on the right side." has been omitted.

17. On page 137, legend to Figure 4,7c,d: "(arrows)" has been deleted from the third line.

18. On page 144, legend to Figure 4.13a: "Bambusa sp." has been changed to "Phyllostachys edulis" in the first line.

19. On page 146, legend to Figure 4.15: "A eudicot stem..." has been changed to "A walnut (Juglans nigra) stem...".

20. On page 157, legend to Figure 5.1b,c: “) (b, c RR Wise)” has been deleted from the last line

21. On page 160, legend to Figure 5.2b: attribution "(Redrawn from Wikipedia)" has been changed to "(Redrawn from Public domain)".

22. On page 160, legend to Figure 5.2c: attribution "(Redrawn from Wikipedia)" has been changed to "(Redrawn from Public domain)".

23. On page 161, legend to Figure 5.2d: attribution "(Redrawn from Wikipedia)" has been changed to "(Redrawn from Public domain)".

24. On page 161, legend to Figure 5.2e: attribution "(Redrawn from Wikipedia)" has been changed to "(Redrawn from Public domain)". 
25. On page 197, legend to Figure 6.6c,d: the attributions at the end of the legend have been changed from "(c MC Ledbetter and KR Porter (1970); d RR Wise) to "(c,d RR Wise)".

26. On page 199, legend to Figure 6.6h: "(SA 3.0)" has been deleted in the first line and the attributions "( $h, \mathbf{i} R$ R Wise)" have been changed to "(h Barbetorte CC SA 3.0; i RR Wise)".

27. On page 222, end of second paragraph: the word "produced" has been inserted in front of "throughout the growing season.".

28. On page 223, legend to Figure 7.4a,b: “; b JD Mauseth, UT Austin" has been deleted from the last line.

29. On page 234, end of the first paragraph, twelfth line: "are an engineering marvel." has been changed to "is an engineering marvel.".

30. On page 235, legend to Figure 7.6 n,o: "(n, o RR Wise)" has been changed to ( $\mathbf{n}$ Jansen et al. 2004; o RR Wise)

31. On page 237, legend to Figure 7.7 a,b: "JM Coulter, CR Barnes and HC Cowles (1910), public domain;" has been deleted. Both images are by RR Wise.

32. On page 238 , legend to Figure $7.7 \mathrm{f}$,g: figure was mislabeled as 7.6. It has been changed to 7.7. Also, in figure legend; the attributions "( $\mathrm{f}, \mathrm{g}$ RR Wise; f from Crang and Vassilyev 2003)" have been changed to "(f from Crang and Vassilyev 2003; g RR Wise)".

33. On page 248, seventh line of the first paragraph of section 8.1: bold font has been used to emphasize "phloem parenchyma”.

34. On page 252, five lines from the bottom of the page: bold font has been removed from "sieve cells".

35. On page 253, legend to Figure 8.2: attribution "(RR Wise)" has been deleted and replaced with "(Redrawn from Crang and Vassilyev 2003)".

36. On page 254, legend to Figure 8.3a:attribution "(JD Curtis, UW Stevens Point)" has been deleted and replaced with "(Redrawn from Crang and Vassilyev 2003)".

37. On page 259, legend to Figure 8.4a-d: attribution “(b, c RR Wise)" has been delete and replaced with "(Redrawn from Crang and Vassilyev 2003)".

38. On page 286, legend to Figure 9.2b: "See below for discussion." has been deleted.

39. On page 321 , legend to Figure 10.1a-d: the attribution “ (a, b, d RR Wise; c F Vincentz, CC BY-SA 3.0)" has been changed to "(a,d RR Wise; b JJ Wise; c c F Vincentz, CC BY-SA 3.0)".

40. On page 323, legend to Figure 10.1 e,f: the bold emphasis has been removed from "e" and "f" from the first sentence of figure legend. That sentence reads, "e, $\mathbf{f}$ Water and ion uptake by plant roots may take e a symplastic pathway (red arrows) or $\mathbf{f}$ an apoplastic pathway (blue arrows) from...."

41. On page 323, legend to Figure 10.1 e,f: attribution "(Modified from F Vincentz, CC BY-SA 3.0)" has been replaced with “(e RR Wise; f redrawn from Crang and Vassilyev 2003)". 
42. On page 325 , third line of the third paragraph: "radical" has been changed to "radicle".

43. On page 338 , second paragraph: the emphasis italic to "tetrarch" has been changed to boldface.

44. On page 357, legend to Fig. 11.1a-c: "a-c" has been added after Fig. 11; and the first line has been modified from "leaves (L), flowers ( $\mathrm{Fl}$ ), and the fruit (Fr) that..." to "leaves, flowers, and the fruit that...".

45. On page 361, legend to Fig. 11.2: "a,b" has been added after Fig.11.2; the first line has been modified from "with node and internode labeled." to "with node labeled."

46. On page 368 , legend to Figure $11.5 \mathrm{c}, \mathrm{d}$ : "c,d" has been added after Fig. 11.5. "calcium oxalate" has been added before "crystals in cortex" to read "calcium oxalate crystals in cortex".

47. On page 369, Fig. 11.5e: "cambium" in the fourth line has been changed to "cambial zone".

48. On page 375, legend for Fig. 11.6: "xylem-phloem" in the first line has been changed to "xylem (X)-phloem (P)".

49. On page 377, legend for Fig. 11.6: "f,g." has been added after Fig. 11.6.

50. On page 386: legend to Fig. 11.9 has been changed to Fig. 11.9 a,b.

51. On page 386: legend to Fig. 11.9 has been changed to Fig. 11.9 c,d.

52. On page 388, legend to Fig. $11.9 \mathrm{~h}$ : at the end of first sentence "(PMT)" has been added after "primary thickening meristem".

53. On page 398: third line in the second paragraph has been changed from "Fig. 14.7b" to "Fig. 14.7b,c".

54. On page 401: Figure legend "Fig. 12.2" has been changed to "Fig. 12.2 c,d".

55. On page 401, sixth line from the bottom of the page: "Fig. 12.2h" has been changed to "Fig. 12.2h,g".

56. On page 405: legend for Fig. 12.2 has been changed to "Fig. $12.2 \mathrm{i}-\mathrm{n}$ ".

57. On page 408: legend for Fig. 12.3 (bottom) has been changed to "Fig. 12. 3 b,c".

58. On page 417: legend for Fig. 12.5 has been changed to "Fig. $12.5 \mathrm{a}, \mathrm{b}$ ".

59. On page 419: legend for Fig. 12.5 has been changed to "Fig. $12.5 \mathrm{c}, \mathrm{d}$ "'

60. On page 424: legend for Fig. 12.7 has been changed to "Fig. 12.7a-c".

61. On page 425: legend for Fig. 12.7 has been changed to "Fig. 12.7e,f".

62. On page 426: text - second to last sentence - "In floating leaves, stomata are found on upper surface exposed to the air..." has been modified to "In floating leaves, stomata are found on the upper surface exposed to the air...".

63. On page 428: legend for Fig. 12.8 has been changed to "Fig. $12.8 \mathrm{~d}, \mathrm{e}$." 
64. On page 436, legend for Fig. 12.10 d: "next years' leaf" in the fourth line has been changed to "next year's leaf".

65. On page 436, in Concept Review 12.1 at the end of the third line: the comma after the word "shapes" has been removed to read "organs, other shapes and functions..."

66. On page 436, in Concept Review 12.2: entire first sentence has been emphasized in italics.

67. On page 445, third line from the bottom of page: "cells, on leaves of the creosote bush,.." has been modified to "cells, such as on leaves of the creosote bush,..."

68. On page 449, legend "Fig. 13.1" was changed to "Fig. 13.1g-h".

69. On page 450, legend Fig. 13.1 j,k: "d" and "e" have been changed to " $j$ " and " $k$ ", respectively, throughout the legend.

70. On page 464, legend "Fig. 13.4e" has been changed to "Fig. 13.4 e,f".

71. On page 467, legend "Fig 13.4" has been changed to "Fig. 13.4 p,q"; and the third line of the legend has been modified from "high levels of calcium (highlighted in yellow) suspended by a silicon-rich stalk (green)" to "high levels of calcium (Ca, highlighted in yellow) suspended by a siliconrich stalk (Si, green)".

72. On page 470, legend to Figure 13.6: attribution "RR Wise" has been changed to "CF Crang".

73. On page 474: Question 3 "Epithelial cells of resin ducts are densely cytoplasmic" has been modified to "Epithelial cells of Apium resin ducts are densely cytoplasmic".

74. On page 474, Question 4: the answers have been modified to start with capital letters: a. Dionaea; b. Dracaena; c. Drosera; d. Drosphyllum; e. Pinguicula.

75. On page 499, legend to Figure 14.9n,o: "Olympia, WA" has been added after " $\mathrm{C}$ Earle" in the attributions.

76. On page 501: legend to Fig. 14.6 has been modified to "Fig. 14.6 b,c".

77. On page 504, in point 14.5: "Cambium" has been modified to "cambia".

78. On page 519, legend to Figure 15.3 i,j: the attributions have been modified from "( $i, j$ RR Wise)" to "(Specimen prepared by JF Reed, Dartmouth College, i, j RR Wise)”.

79. On page 524, first paragraph, sixth line: the text, “...or in extant species such as the firs (Abies sp.) that are considered to have primitive features (Carlquist 2001). Lacking the ability to produce resin in the xylem, fir wood has poor resistance..." has been modified to "...or in extant species such as the true firs (Abies sp.) that are considered to have primitive features (Carlquist 2001). Lacking the ability to produce resin in the xylem, balsam fir wood has poor resistance...".

80. On page 538, legend to Figure 15.9c-e: "d Cross and e longitudinal section..." has been changed to "d Longitudinal and e cross sections..." 
81. On page 551: the webpage address in Otaigbe reference has been modified from "Online: wwwnsfgov/news/speaches/ Nov.19, 2014" to "Online: www.nsf.gov."

82. On page 563: the third line of legend to Figure $16.4 \mathrm{~b}$ has been changed from "(red box to the left)" to "(red box to the right)".

83. On page 574: answers to question \#9 have been modified to start with capital letters: a. Robinia, i. Betula, c Quercus, d. Tilia, e. Quercus

84. On page 583 , the text "...if the ovary is above the whorls, it is termed superior or hypogynous (Fig. 17.1i). If the whorls are attached midway, the position is half inferior or perigynous (Fig. 17.1j). If the whorls are attached at the top of the ovary, leaving it beneath, the ovary is termed inferior or epigynous (Fig. 17.1k)." has been modified to "...if the whorls are attached at the top of the ovary, leaving it beneath, the ovary is termed inferior or epigynous (Fig. 17.1i). If the whorls are attached midway, the position is half inferior or perigynous (Fig. 17.1j). If the ovary is above the whorls, it is termed superior or hypogynous (Fig. 17.1k).”.

85. On page 598, legend to Figure 17.5a: "exine (E)" in the fourth line has been changed to "exine (E1)"; and "cell wall (CW)" in the $6^{\text {th }}$ line has been changed to "cell wall (CW1)".

86. On page 601, legend to Figure 17.6a-f: "a-f" has been added in the attributions to read "(a-f RR Wise)"

87. On page 619 , legend to Fig. 18.2 a-c: " $a$ androecium (stamens), $p$ petals, $s$ sepal, $r$ receptacle, and $g$ gynoecium" has been modified to " $A$ androecium (stamens), $P$ petals, $S$ sepal, $R$ receptacle, and $G$ gynoecium".

88. On page 624, second to last line: text "An overview of megasporogenesis in given in" has been modified to "An overview of megasporogenesis is given in".

89. On page 625, Figure $18.2 \mathrm{~g}$ : on the figure, "Chalaze" has been changed to "Chalaza".

90. On page 625, Figure $18.3 \mathrm{~h}$-j: the word "Embryo" has been deleted from the figure.

91. On page 631, legend to Figure 18.4 q: “(P)” has been added after "lower polar nucleus" in the fifth line.

92. On page 639 in text: the first word of the third line "ten" has been changed to "tend".

93. On page 646, Question \#3: "these terms can be used interchangeable" has been modified to "these terms can be used interchangeably".

94. On page 657, at the end of the first paragraph: "Fig. 19i, k" has been changed to "Fig. 19i,j"

95. On page 663: "Examples of aggregate fruits include strawberries (achene), magnolia (follicles, samaras, or berries), blackberry and raspberry (drupelets)." has been changed to "Examples of aggregate fruit include strawberries (achene), custard apple (berries), raspberry (drupes) and velvet leaf (follicles) (Fig. 19.5a-d)... 
96. On page 663, legend to Figure 19.5a-d: "(S Lyons-Sobaski)" has been removed from the fourth line and "(Lli324, CC0)" has been removed from the fifth line, since the attributions were already available at the end of the legend.

97. On page 665, fourth line from the bottom of paragraph: "have seeds that contain well-developed plumule" has been modified to "have seeds that contain a well-developed plumule".

98. On page 665, legend to Fig. 19.6a: "(SA)" and "(E)" from the second and third line of the legend have been deleted since they were unavailable in the figure.

99. On page 666, legend to Fig. 19.6c: "(Sc)" in the fourth line has been changed to "(S)".

100. On page 690, Ch. 13, Concept Assessment: answer to \#4 has been modified to "a" from "d".

101. On page 690, Ch. 13, Concept Assessment: answer to \#5 has been modified to " $e$ " from " $b$ ".

102. On page 690, Ch. 13, Concept Assessment: answer to \#7 has been modified to " $c$ " from "a".

103. On page 690, Ch. 13, Concept Assessment: answer to \#8 has been modified to "a" from " $c$ ".

104. On page 690, Ch. 13, Concept Assessment: answer to \#9 has been modified to "d" from "c".

105. On page 690, Ch. 13, Concept Assessment: answer to \#11 has been modified to "b" from "a".

106. On page 694: numbers to answers at the end of chapter questions should go from 1 to 13 .

107. On pages 144 and 363: an index entry "Phyllostachys edulis" has been added. 